\title{
Crop yield, $P$ uptake and soil organic phosphorus fractions in response to short-term tillage and fertilization under a rape- rice rotation in central China
}

\author{
Z. S. Zhang, C.G. Cao, M. L.Cai, C.F. Li* \\ MOA Key Laboratory of Crop Ecophysiology and Farming System in the Middle Reaches of the Yangtze River, P.R. \\ China / College of Plant Science and Technology, Huazhong Agricultural University, Wuhan 430070, P.R. China. \\ *Corresponding author: lichengfang@mail.hzau.edu.cn
}

\begin{abstract}
We conducted a 3-year field experiment on an Anthrosol paddy soil to investigate changes in crop yield, P uptake and soil organic phosphorus (P) fractions after 3 years of conventional tillage (CT) conversion to no-tillage (NT) under a rape - rice rotation in central China. Treatments were established following a split-plot design of a randomized complete block with tillage practice as the main plot and fertilizer as the sub-plot treatment. The yields of rape and rice ranged from 1378 to $2264 \mathrm{~kg} \mathrm{ha}^{-1}$ and from 5895 to $9453 \mathrm{~kg} \mathrm{ha}^{-1}$ across 3 years, respectively. Moreover, P uptake for rape and rice (aboveground) varied from 3.9 to $10.4 \mathrm{~kg} \mathrm{ha}^{-1}$ and from 9.5 to $32.0 \mathrm{~kg} \mathrm{ha}^{-1}$, respectively. Fertilization significantly enhanced crop yields and $\mathrm{P}$ uptake, but tillage did not affect the yields and $\mathrm{P}$ uptake. Fertilization significantly increased total $\mathrm{P}$ concentrations, acid phosphatase activity, Bray-1 P and labile organic $\mathrm{P}$ in the $0-5 \mathrm{~cm}$ soil layer. Compared to the CT treatments, the NT treatments had significantly higher acid phosphatase activity, total $\mathrm{P}$, Bray-1 P, total organic $\mathrm{P}$ and organic $\mathrm{P}$ fractions in the $0-5 \mathrm{~cm}$ soil layer but lower organic P fractions in the 5-20 cm soil layer. Therefore, our results suggest that short-term NT does not enhance organic $\mathrm{P}$ concentrations in the $0-20 \mathrm{~cm}$ soil layer, and only improve $\mathrm{P}$ availability on the soil surface.
\end{abstract}

Keywords: Fertilization, organic P fractions, P uptake, tillage practices, yield

\section{Introduction}

Phosphorus is one of the major nutrients limiting agricultural production in China, where soil deficient in $\mathrm{P}$ constitutes one-third of the cultivated land (Bao, 2000). Hence, chemical P fertilizers are added to agricultural soils to improve crop production. However, excess application of $\mathrm{P}$ fertilizers is common and this poses a threat to the quality of surface water within the watershed (Daniel et al.
1998). Soil organic $P$ accounts for a significant portion of total P, ranging from $20 \%$ to $80 \%$ in most soils (Dalal et al., 1997), and contributes substantially to plant-available P through mineralization (Sharpley, 1985). Therefore, Sharpley (1985) suggested that it is essential to include inorganic and organic $\mathrm{P}$ in soil $\mathrm{P}$ fertility tests for better prediction of plant responses. 
Because soil organic P pool is a heterogeneous mixture of organic substances, different fractions of soil organic P may have different effects on soil fertility and quality. $\mathrm{P}$ fractionation has been proven useful in identifying soil P pools in various ecosystems (Sharpley, 1985). The fractionation scheme developed by Bowman and Cole (1978) facilitates separation of soil organic P into four distinct fractions of varying lability: labile organic $\mathrm{P}$, moderately labile organic $\mathrm{P}$, moderately resistant organic $\mathrm{P}$ and highly resistant organic $\mathrm{P}$. According to Conte et al. (2003), the concentration of labile organic $\mathrm{P}$ and moderately labile organic $\mathrm{P}$ determined by the method of Bowman and Cole (1978) is a useful index of whether a particular soil is rich in available organic P. Furthermore, Bao (2000) suggested that the method proved very useful in quantifying soil organic $P$ pools of different availability and is widely used to study effects of fertilizer and management practices on the soil $\mathrm{P}$ status in field experiments.

Conventional tillage (CT) mixes soils with crop residues, thus accelerating residue decomposition. In addition, tilling soil disrupts soil aggregates, resulting in concomitant organic matter oxidation, thus affecting availability and distribution of soil organic $\mathrm{P}$ (Selles et al., 1997). However, there was no consensus on soil organic P between NT and CT. For example, Essington and Howard (2000) reported that NT had significantly greater organic P than CT. However, Selles et al. (1997) indicated that the concentration of organic $\mathrm{P}$ in untilled soils increased at the $0-6 \mathrm{~cm}$ depth, and decreased at the lower depth when compared to tilled soils. In addition, Huang and Yuan (1994) found a decrease in organic $\mathrm{P}$ under paddy soils. Hence, more information about tillage practices effects on organic $\mathrm{P}$, especially on different organic $\mathrm{P}$ fractions, is essential to understand soil $\mathrm{P}$ dynamics and to determine if transformations among organic $\mathrm{P}$ fractions are related to $\mathrm{P}$ availability.

Chemical P fertilizers applied to soils can been taken up by microbes to form organic $\mathrm{P}$ compounds present in microbial detritus (Lee et al., 2004). Moreover, fertilization increases crop biomass and in turn more cop residues are added to soils, thus the formation of organic P. fractions Lan et al. (2012) and Lee et al. (2004) reported that fertilizer applied for many years resulted in accumulation of organic $\mathrm{P}$ fractions in paddy soils. However, Zhang et al. (2006) did not observe significant changes in paddy soil organic P fractions due to application of chemical fertilizer. Therefore, more knowledge on the effect of fertilization on organic $\mathrm{P}$ fractions of paddy soils is needed.

Conventional tillage in agricultural production systems affects physical, chemical, and biological properties (Quan et al., 2005), thus affecting crop yields and $\mathrm{P}$ uptake. It was reported that the differences in crop yields between tillage systems are affected by the duration time of NT. Sharma et al. (2005) reported that short-term NT $(<4$ years $)$ significantly reduced crop yields in rice-based cropping systems. However, Qin et al. (2010) found similar rice yields between tillage treatments in a 2-year field experiment. Wang et al. (2001) indicated that NT had higher rice yields compared with $\mathrm{CT}$ in an 8-year field experiment. Therefore, the results obtained were often contradictory and inconclusive possibly due to variability in soil type, cropping systems, and climate and further research on effects of the duration time of NT on crop yields is still required.

Central China is a major rice producing region accounting for $70 \%$ of total rice production in China (HPBS, 2010). Recently, NT has become increasingly popular in the region, encompassing about 150,000 ha in 2009 (HPBS, 2010). To our knowledge, little information is available about effects of short-term tillage and chemical fertilizer application on crop yield, $\mathrm{P}$ uptake and soil organic $\mathrm{P}$ fractions under a rape-rice rotation in this region. Therefore, the objectives of this paper were (1) to investigate crop yields and $\mathrm{P}$ uptake, and (2) to quantify the concentrations of total P, labile organic P, moderately labile organic P, moderately resistant organic $\mathrm{P}$ and highly resistant organic $\mathrm{P}$ after 3 years of $\mathrm{CT}$ conversion to NT under a rape - rice rotation in central China. 


\section{Materials and Methods}

\subsection{Site description}

The experimental site is located at the Experimental Farm at Zhonggui Country, Dafashi Town, Wuxue City, Hubei Province, China (295 $\left.55^{\prime} \mathrm{N}, 115^{\circ} 30^{\prime} \mathrm{E}\right)$. This region has a humid mid-subtropical monsoon climate and most of the rainfall occurred between April and August. From 2006 to 2009, the rainfall ranged from 1426 to $1546 \mathrm{~mm}$ and annual mean air temperature varied from $24.7^{\circ} \mathrm{C}$ to $25.7^{\circ} \mathrm{C}$. The soil at the experiment site is a clay loam soil and is classified as an Anthrosol. The main soil properties $(0-20 \mathrm{~cm}$ depth) of the site sampled in October 2006 were as follows: $\mathrm{pH}_{\mathrm{H} 2 \mathrm{O}}, 6.5$; organic $\mathrm{C}, 18.3 \mathrm{~g} \mathrm{~kg}^{-1}$; total $\mathrm{N}, 1.1$ $\mathrm{g} \mathrm{kg}^{-1} ; \mathrm{NO}_{3}^{-}-\mathrm{N}, 1.0 \mathrm{mg} \mathrm{kg}^{-1} ; \mathrm{NH}_{4}^{+}-\mathrm{N}, 1.9 \mathrm{mg} \mathrm{kg}^{-1}$; total $\mathrm{P}, 0.7 \mathrm{~g} \mathrm{~kg}^{-1}$; Bray 1-P, $3.7 \mathrm{mg} \mathrm{kg}^{-1}$; labile organic $\mathrm{P}$, $1.9 \mathrm{mg} \mathrm{kg}^{-1}$; moderately labile organic P, $212 \mathrm{mg} \mathrm{kg}$ ${ }^{1}$; moderately resistant organic $\mathrm{P}, 24.9 \mathrm{mg} \mathrm{kg}^{-1}$; highly resistant organic $\mathrm{P}, 40.8 \mathrm{mg} \mathrm{kg}^{-1}$; and $\mathrm{CH}_{3} \mathrm{COONH}_{4}$ extractable $\mathrm{K}, 111 \mathrm{mg} \mathrm{kg}^{-1}$.

\subsection{Experimental Design}

The experimental site was cultivated with a rape-rice rotation for 30 years prior to October 2006, where rice was transplanted with $\mathrm{CT}$ from May to October each year and rape was planted with CT from October to May the following year. The experiment was established in October 2006, three years prior to study initiation and is designed following a split-plot design of a randomized complete block with tillage practices [no-tillage (NT) and conventional tillage (CT)] in the main plot and fertilizers $[0$ and application of chemical NPK fertilizer] in the sub-plots. There were four treatments including: NT0, CT0, NTF and CTF. Each treatment had three replications. Each plot was isolated with a plastic film driven to a depth of $40 \mathrm{~cm}$ along the inner edge of the field ridge $(30 \mathrm{~cm}$ at the base and $30 \mathrm{~cm}$ in height) in order to prevent lateral water movement caused by either leakage or permeable lateral flow. Each plot had an area of $45 \mathrm{~m}^{2}$ and an inlet for irrigation as well as an outlet for drainage.
For the NT plots, no soil disturbance occurred except for herbicide (glyphosate) application, direct sowing, and fertilizer application. Immediately after rape or rice was harvested, the $\mathrm{CT}$ plots were tilled by hand to a $10 \mathrm{~cm}$ depth, then moldboard plowed to a depth of $20 \mathrm{~cm}$ using a SNH554 tractor (Shanghai New Holland Agriculture Machinery Co., Ltd.). Before rape planting or rice sowing (about May or October), the CT plots were disked and moldboard plowed for weed control and bedding; this was followed by an application of fertilizer. The rape and rice were harvested in May and October in each year. For fertilized treatments, chemical inorganic P fertilizers were broadcast just before sowing or planting, with rape receiving $39 \mathrm{~kg} \mathrm{P}$ $\mathrm{ha}^{-1}$ and rice receiving $59 \mathrm{~kg} \mathrm{P} \mathrm{ha}^{-1}$. Additional $\mathrm{N}$ and $\mathrm{K}$ fertilizers were applied, with rape receiving $180 \mathrm{~kg} \mathrm{~N}$ $\mathrm{ha}^{-1}$ and $124 \mathrm{~kg} \mathrm{~K} \mathrm{ha}^{-1}$, respectively and rice receiving $210 \mathrm{~kg} \mathrm{~N} \mathrm{ha}^{-1}$ and $199 \mathrm{~kg} \mathrm{~K}_{2} \mathrm{O} \mathrm{ha}^{-1}$, respectively. Rice seeds were sown manually at a rate of $22.5 \mathrm{~kg} \mathrm{ha}^{-1}$ and rape seedlings were transplanted at a planting density of $33 \mathrm{~cm} \times 16 \mathrm{~cm}$ with one seedling per hill. The mid-season rice variety was Liangyoupeijiu (Oryza sativa L.) and the rape variety was Huayouzaliuhao (Brassica napus).

\subsection{Soil sampling and analytical methods}

Soil samples ( $5 \mathrm{~cm}$ diameter) were collected using a soil sampler with a diameter of $5 \mathrm{~cm}$ at eight random positions in each plot on October 8, 2009 (just after rice harvesting). Samples were sectioned into 0-5 $\mathrm{cm}, 5-10 \mathrm{~cm}$ and $15-20 \mathrm{~cm}$ depth increments. Total $\mathrm{P}\left(\mathrm{g} \mathrm{kg}^{-1}\right)$ in soil and crop plant were digested at 300 by adding concentrated $\mathrm{H}_{2} \mathrm{SO}_{4}$ and $\mathrm{HClO}_{4}$ (Bao, 2000), and analyzed at $700 \mathrm{~nm}$ using 721 Visible Spectrophotometer (Jinhua Instruments, Shanghai) according to the method of Murphy and Riley (1962). The available P (Bray-1 P) content was extracted by adding $\mathrm{HCl}$ and $\mathrm{NH}_{4} \mathrm{~F}$, and determined using the Visible Spectrophotometer by the method of Bray and Kurtz (1945). Acid phosphatase enzyme activity was estimated by measuring the release of $p$-nitrophenol from $p$-nitrophenyl phosphate following exposure to soil in a modified universal buffer (MUB) at $\mathrm{pH}$ 
6.5, as described by Tabatabai and Bremner (1969). Soil samples $(1 \mathrm{~g})$ were incubated with $1 \mathrm{~mL} 0.1 \mathrm{M}$ p-nitrophenyl phosphate and $4 \mathrm{~mL}$ MUB for $60 \mathrm{~min}$ at 37. At the end of the incubation period, $1 \mathrm{~mL} 0.5 \mathrm{M} \mathrm{CaCl}_{2}$ and $4 \mathrm{~mL} 0.5 \mathrm{M} \mathrm{NaOH}$ were added. After, the solution was filtered quickly. Samples were homogenized and the $p$-nitrophenol formed was determined by the Visible Spectrophotometer at $400 \mathrm{~nm}$.

Soil organic $\mathrm{P}$ was separated into four fractions according to the method of Bowman and Cole (1978), including (1) labile organic P, extracted with $0.5 \mathrm{M} \mathrm{NaHCO}_{3}(\mathrm{pH} 8.5)$, with organic $\mathrm{P}$ determined by the increase in inorganic $\mathrm{P}$ after perchloric acid digestion; (2) moderately labile organic $\mathrm{P}$, extracted with $1.0 \mathrm{M} \mathrm{H}_{2} \mathrm{SO}_{4}$, with organic $\mathrm{P}$ determined as for labile organic P; (3) moderately resistant and (4) highly resistant organic $\mathrm{P}$, determined by transferring the $1.0 \mathrm{M} \mathrm{H}_{2} \mathrm{SO}_{4}$-extracted soil samples into filter paper, then washing the samples with $3 \mathrm{ml}$ ethanol, followed by drying and extraction with $0.5 \mathrm{M}$ $\mathrm{NaOH}$. These organic $\mathrm{P}$ fractions were determined together after perchloric acid digestion (fraction I); then an aliquot of the extract was acidified with concentrated hydrochloric acid to $\mathrm{pH} 1-1.5$, and moderately resistant organic $\mathrm{P}$ was determined after perchloric acid digestion (fraction II), with highly resistant organic $\mathrm{P}$ determined by subtracting fraction I from fraction II. P was determined according to the method of Murphy and Riley (1962). Total organic P was calculated as the sum of four organic P fractions, and total inorganic $\mathrm{P}$ was calculated as the difference between total $\mathrm{P}$ and total organic P. All soil samples were analyzed in triplicate.

\subsection{Analysis of crop grain yield and P uptake by crops}

Rape or rice plants (aboveground part) at harvesting were collected at three random positions in each plot by using a $1 \mathrm{~m} \times 1 \mathrm{~m}$ frame; rape and rice plants were threshed, and the seeds of rape and grains of rice were adjusted to moisture contents of $9 \%$ and $14 \%$, respectively. The crop yield was recorded and the aboveground plants (grain and straw) were dried in an oven at 85 and then rape or rice (aboveground) was determined as the product of the concentration of total P and dry weight of crop (aboveground).

\subsection{Data analysis}

The SPSS 16.0 analytical software package was used for all statistical analyses. We used two-way ANOVA of SPSS 16.0 to determine effects of tillage or fertilization on crop yields and soil P fractions. Individual means were compared to the L.S.D. test. Only means statistically different at $p \leq 0.05$ were considered different and the significance probability levels of the results are given at the $p<0.05(*)$ and $p<0.01(* *)$ levels. Regression models were fitted to the data in an effort to describe the relationships between soil organic $\mathrm{P}$ fractions and rice yields.

\section{Results}

The yields of rape and rice significantly varied across three years and the yields of rape and rice ranged from 1378 to $2264 \mathrm{~kg} \mathrm{ha}^{-1}$, and from 5895 to $9453 \mathrm{~kg} \mathrm{ha}^{-1}$ across three years, respectively (Table 1). Application of fertilizer significantly increased yields of rape by $30 \%-42 \%$ and rice by $20 \%-58 \%$. Although tillage practices did not significantly affect grain yields of rape or rice, the NT treatments slightly increased the yields of rape and rice compared to the $\mathrm{CT}$ treatments across three years generally. As Table 1 shows, $\mathrm{P}$ uptake for rape and rice (aboveground) ranged from 3.9 to $10.4 \mathrm{~kg} \mathrm{ha}^{-1}$ and from 9.5 to $32.0 \mathrm{~kg} \mathrm{ha}^{-1}$ across three years, respectively.

Fertilization significantly increased the P uptake by 2.22.3 times for rape and 2.5-3.4 times for rice compared to no fertilizer application. Although tillage practices did not affect $\mathrm{P}$ uptake for rape or rice, the NT treatments slightly increased $\mathrm{P}$ uptake for rape and rice compared to the CT treatments across three years generally. There was also no significant interaction of tillage and fertilizer on grain yield or P uptake by crop plants. 
Table 1. Yields of rape and rice $\left(\mathrm{kg} \mathrm{ha}^{-1}\right)$ and $\mathrm{P}$ uptake by rape and rice $\left(\mathrm{kg} \mathrm{P} \mathrm{ha}^{-1}\right)$ under different treatments after 3 years of rape-rice rotation

\begin{tabular}{|c|c|c|c|c|c|c|}
\hline \multirow{3}{*}{$\begin{array}{l}\text { Treatment } \\
\mathrm{s}\end{array}$} & \multicolumn{6}{|l|}{ Rape } \\
\hline & \multicolumn{2}{|l|}{2007} & \multicolumn{2}{|l|}{2008} & \multicolumn{2}{|l|}{2009} \\
\hline & $\begin{array}{l}\text { Grain } \\
\text { yield }\end{array}$ & $\begin{array}{l}\text { P uptake } \\
\text { (aboveground } \\
\text { plant) }\end{array}$ & $\begin{array}{l}\text { Grain } \\
\text { yield }\end{array}$ & $\begin{array}{l}\text { P uptake } \\
\text { (aboveground } \\
\text { plant) }\end{array}$ & $\begin{array}{l}\text { Grain } \\
\text { yield }\end{array}$ & $\begin{array}{l}\text { P uptake } \\
\text { (aboveground } \\
\text { plant) }\end{array}$ \\
\hline NT0 & $\begin{array}{l}1405 \mathrm{~B} \\
(112)\end{array}$ & $\begin{array}{l}4.0 \mathrm{~B} \\
(0.4)\end{array}$ & $\begin{array}{l}1378 \mathrm{~B} \\
(153)\end{array}$ & $\begin{array}{l}3.9 \mathrm{~B} \\
(0.4)\end{array}$ & $\begin{array}{l}1553 \mathrm{~B} \\
(154)\end{array}$ & $\begin{array}{l}4.3 \mathrm{~B} \\
(0.4)\end{array}$ \\
\hline СТ0 & $\begin{array}{l}1456 \mathrm{~B} \\
(98)\end{array}$ & $\begin{array}{l}4.1 \mathrm{~B} \\
(0.5)\end{array}$ & $\begin{array}{l}1479 \mathrm{~B} \\
(123)\end{array}$ & $\begin{array}{l}4.1 \mathrm{~B} \\
(0.6)\end{array}$ & $\begin{array}{l}1541 \mathrm{~B} \\
(163)\end{array}$ & $\begin{array}{l}4.5 \mathrm{~B} \\
(0.4)\end{array}$ \\
\hline NTF & $\begin{array}{l}1989 \mathrm{~A} \\
(156)\end{array}$ & $\begin{array}{l}9.1 \mathrm{~A} \\
(1.0)\end{array}$ & $\begin{array}{l}1948 \mathrm{~A} \\
(94)\end{array}$ & $\begin{array}{l}8.9 \mathrm{~A} \\
(1.2)\end{array}$ & $\begin{array}{l}2171 \mathrm{~A} \\
(215)\end{array}$ & $\begin{array}{l}9.9 \\
(0.9)\end{array}$ \\
\hline $\mathrm{CTF}$ & $\begin{array}{l}2042 \mathrm{~A} \\
(173)\end{array}$ & $\begin{array}{l}9.3 \mathrm{~A} \\
(0.8)\end{array}$ & $\begin{array}{l}1925 \mathrm{~A} \\
(120)\end{array}$ & $\begin{array}{l}8.8 \mathrm{~A} \\
(1.2)\end{array}$ & $\begin{array}{l}2264 \mathrm{~A} \\
(115)\end{array}$ & $\begin{array}{l}10.4 \mathrm{~A} \\
(1.7)\end{array}$ \\
\hline \multicolumn{7}{|c|}{ Analysis of variance } \\
\hline $\mathrm{F}$ & $* *$ & $* *$ & $* *$ & $* *$ & $* *$ & $* *$ \\
\hline $\mathrm{T}$ & ns & ns & ns & ns & ns & ns \\
\hline $\mathrm{F} \times \mathrm{T}$ & ns & ns & ns & ns & ns & ns \\
\hline \multirow{3}{*}{$\begin{array}{l}\text { Treatment } \\
\mathrm{s}\end{array}$} & \multicolumn{6}{|l|}{ Rice } \\
\hline & \multicolumn{2}{|l|}{2007} & \multicolumn{2}{|l|}{2008} & \multicolumn{2}{|l|}{2009} \\
\hline & $\begin{array}{l}\text { Grain } \\
\text { yield }\end{array}$ & $\begin{array}{l}\text { P uptake } \\
\text { (aboveground } \\
\text { plant) }\end{array}$ & $\begin{array}{l}\text { Grain } \\
\text { yield }\end{array}$ & $\begin{array}{l}\text { P uptake } \\
\text { (aboveground } \\
\text { plant) }\end{array}$ & $\begin{array}{l}\text { Grain } \\
\text { yield }\end{array}$ & $\begin{array}{l}\text { P uptake } \\
\text { (aboveground } \\
\text { plant) }\end{array}$ \\
\hline NT0 & $\begin{array}{l}5895 \mathrm{~B} \\
(355)\end{array}$ & $\begin{array}{l}9.5 \mathrm{~B} \\
(1.2)\end{array}$ & $\begin{array}{l}\text { 6521 B } \\
(244)\end{array}$ & $\begin{array}{l}10.5 \mathrm{~B} \\
(1.0)\end{array}$ & $\begin{array}{l}6503 \mathrm{C} \\
(192)\end{array}$ & $\begin{array}{l}10.0 \mathrm{~B} \\
(1.4)\end{array}$ \\
\hline СТ0 & $\begin{array}{l}6020 \mathrm{~B} \\
(416)\end{array}$ & $\begin{array}{l}9.5 \mathrm{~B} \\
(1.0)\end{array}$ & $\begin{array}{l}6556 \mathrm{~B} \\
(186)\end{array}$ & $\begin{array}{l}10.4 \mathrm{~B} \\
(1.5)\end{array}$ & $\begin{array}{l}6713 \mathrm{C} \\
(113)\end{array}$ & $\begin{array}{l}10.3 \mathrm{~B} \\
(1.6)\end{array}$ \\
\hline NTF & $\begin{array}{l}9307 \mathrm{~A} \\
(552)\end{array}$ & $\begin{array}{l}29.9 \mathrm{~A} \\
(3.9)\end{array}$ & $\begin{array}{l}8090 \mathrm{~A} \\
(156)\end{array}$ & $\begin{array}{l}26.0 \mathrm{~A} \\
(2.4)\end{array}$ & $\begin{array}{l}7830 \mathrm{~B} \\
(110)\end{array}$ & $\begin{array}{l}25.4 \mathrm{~A} \\
(2.2)\end{array}$ \\
\hline CTF & $\begin{array}{l}9453 \mathrm{~A} \\
(596)\end{array}$ & $\begin{array}{l}32.0 \mathrm{~A} \\
(3.5)\end{array}$ & $\begin{array}{l}8807 \mathrm{~A} \\
(114)\end{array}$ & $\begin{array}{l}29.8 \mathrm{~A} \\
(2.8)\end{array}$ & $\begin{array}{l}8976 \mathrm{~A} \\
(55)\end{array}$ & $\begin{array}{l}28.7 \mathrm{~A} \\
(1.8)\end{array}$ \\
\hline \multicolumn{7}{|c|}{ Analysis of variance } \\
\hline $\mathrm{F}$ & $* *$ & $* *$ & $* *$ & $* *$ & $* *$ & $* *$ \\
\hline $\mathrm{T}$ & ns & ns & ns & ns & ns & ns \\
\hline $\mathrm{F} \times \mathrm{T}$ & ns & ns & ns & ns & ns & ns \\
\hline
\end{tabular}

Different capital letters in a column mean significant difference at the $5 \%$ level between treatments. Values in brackets are standard deviation. NT0, no-tillage with no fertilizer; CT0, conventional tillage with no fertilizer; NTF, no-tillage with application of chemical fertilizer; CTF, conventional tillage with application of chemical fertilizer; F, application of chemical fertilizers; T, tillage practices; ns, not significant; ${ }^{* *}, p<0.01$.

No-tillage (NT) treatments exhibited a strong stratification of acid phospatase activity and the concentrations of soil total $\mathrm{P}$, total inorganic $\mathrm{P}$ and Bray-1 P (Table 2). Compared to the CT treatments, the NT treatments significantly increased acid phospatase activity by $25 \%-30 \%$, total $\mathrm{P}$ concentration by $9 \%$ $10 \%$, total inorganic $\mathrm{P}$ concentration by $4 \%-5 \%$ and
Bray-1 P concentration by $41 \%-45 \%$ the $0-5 \mathrm{~cm}$ soil layer. In the same soil layer, similar to effects of tillage practices, fertilization increased acid phospatase activity by $15 \%-20 \%$, total $\mathrm{P}$ concentration by $9 \%$ $10 \%$, total inorganic P concentration by $16 \%-19 \%$ and Bray-1 P concentration by $26 \%-29 \%$ compared to no fertilizer application. 
Table 2. Concentrations of soil total $\mathrm{P}\left(\mathrm{g} \mathrm{kg}^{-1}\right)$, total inorganic $\mathrm{P}\left(\mathrm{mg} \mathrm{kg}^{-1}\right)$ and Bray-1 $\mathrm{P}\left(\mathrm{mg} \mathrm{kg}^{-1}\right)$, and acid phosphatase activity $\left(\mathrm{mg} \mathrm{TPF} \mathrm{kg}^{-1} \mathrm{~h}^{-1}\right)$ under different treatments after 3 years of rape-rice rotation

\begin{tabular}{|c|c|c|c|c|c|c|c|c|c|c|c|c|}
\hline \multirow[t]{2}{*}{ Treatments } & \multicolumn{3}{|l|}{ Total P } & \multicolumn{3}{|c|}{ Total inorganic $\mathrm{P}$} & \multicolumn{3}{|l|}{ Bray-1 P } & \multicolumn{3}{|c|}{ Acid phosphatase activity } \\
\hline & $0-5 \mathrm{~cm}$ & $0-5 \mathrm{~cm}$ & $0-5 \mathrm{~cm}$ & $0-5 \mathrm{~cm}$ & $0-5 \mathrm{~cm}$ & $0-5 \mathrm{~cm}$ & $0-5 \mathrm{~cm}$ & $0-5 \mathrm{~cm}$ & $0-5 \mathrm{~cm}$ & $0-5 \mathrm{~cm}$ & $0-5 \mathrm{~cm}$ & $0-5 \mathrm{~cm}$ \\
\hline \multirow[t]{2}{*}{ NT0 } & $0.8 \mathrm{aB}$ & $0.7 \mathrm{bA}$ & $0.6 \mathrm{cA}$ & $505 \mathrm{aB}$ & $478 \mathrm{aB}$ & $428 \mathrm{bB}$ & $10.3 \mathrm{aB}$ & $6.9 \mathrm{bA}$ & $5.2 \mathrm{cB}$ & $1.3 \mathrm{aAB}$ & $1.1 \mathrm{bA}$ & $0.6 \mathrm{cA}$ \\
\hline & $(0.06)$ & $(0.07)$ & $(0.05)$ & $(0.07)$ & $(0.06)$ & $(0.04)$ & $(1.3)$ & (1.4) & (1.1) & $(0.4)$ & $(0.3)$ & $(0.1)$ \\
\hline \multirow[t]{2}{*}{ CT0 } & $0.7 \mathrm{aC}$ & $0.7 \mathrm{aA}$ & $0.7 \mathrm{aA}$ & $484 \mathrm{aB}$ & $515 \mathrm{aB}$ & $0.5 \mathrm{aA}$ & $7.3 \mathrm{aD}$ & $7.2 \mathrm{aB}$ & $6.9 \mathrm{aA}$ & $1.0 \mathrm{aC}$ & $1.0 \mathrm{aA}$ & $0.8 \mathrm{bA}$ \\
\hline & $(0.05)$ & $(0.04)$ & $(0.05)$ & $(0.05)$ & $(0.07)$ & $(0.06)$ & $(1.1)$ & (1.4) & (1.1) & $(0.1)$ & $(0.2)$ & $(0.2)$ \\
\hline \multirow[t]{2}{*}{ NTF } & $0.9 \mathrm{aA}$ & $0.8 \mathrm{bA}$ & $0.7 \mathrm{cA}$ & $589 \mathrm{aA}$ & $568 \mathrm{aA}$ & $519 \mathrm{bA}$ & $13.3 \mathrm{aA}$ & $7.8 \mathrm{bB}$ & $6.8 \mathrm{cA}$ & $1.5 \mathrm{aA}$ & $1.0 \mathrm{bA}$ & $0.6 \mathrm{cA}$ \\
\hline & $(0.05)$ & $(0.07)$ & $(0.08)$ & $(0.09)$ & $(0.08)$ & $(0.03)$ & (2.3) & $(1.0)$ & (1.1) & $(0.2)$ & $(0.2)$ & $(0.1)$ \\
\hline \multirow[t]{2}{*}{ CTF } & $0.8 \mathrm{aB}$ & $0.8 \mathrm{aA}$ & $0.7 \mathrm{aA}$ & $561 \mathrm{aA}$ & $573 \mathrm{aA}$ & $512 \mathrm{aA}$ & $9.2 \mathrm{aC}$ & $7.0 \mathrm{bB}$ & $7.1 \mathrm{bA}$ & $1.2 \mathrm{aB}$ & $1.0 \mathrm{aA}$ & $0.6 \mathrm{bA}$ \\
\hline & $(0.09)$ & $(0.08)$ & $(0.09)$ & $(0.08)$ & $(0.07)$ & $(0.05)$ & (2.1) & (1.6) & (2.0) & $(0.3)$ & $(0.2)$ & $(0.1)$ \\
\hline \multicolumn{13}{|l|}{ ANOVA } \\
\hline $\mathrm{F}$ & $* *$ & ns & ns & $* *$ & $* *$ & ns & ** & ns & ns & $*$ & ns & ns \\
\hline $\mathrm{T}$ & * & ns & ns & * & ns & ns & $* *$ & ns & ns & $* *$ & ns & ns \\
\hline $\mathrm{F} \times \mathrm{T}$ & ns & ns & ns & ns & ns & ns & * & ns & ns & ns & ns & ns \\
\hline
\end{tabular}

Different small letters in a line at different soil depths indicate significant difference at the $5 \%$ level. Different capital letters in a column mean significant difference at the $5 \%$ level between treatments. Values in brackets are standard deviation. NT0, no-tillage with no fertilizer; $\mathrm{CT} 0$, conventional tillage with no fertilizer; NTF, no-tillage with application of chemical fertilizer; CTF, conventional tillage with application of chemical fertilizer; F, application of chemical fertilizer; T, tillage practices; ns, not significant; $*, p<0.05 ; * *, p<0.01$.

There was no significant effect of tillage or fertilizer on total $\mathrm{P}$ concentration, Bray-1 $\mathrm{P}$ concentration and acid phospatase activity in the $5-20 \mathrm{~cm}$ soil layer.

Fertilization had no significant effect on total organic P, moderately labile organic P, moderately resistant organic $\mathrm{P}$, and highly resistant organic $\mathrm{P}$ across all depths but significantly increased labile organic P concentration by $19 \%-104 \%$ in the $0-20 \mathrm{~cm}$ soil layer (Table 3). The NT treatments significantly enhanced the concentrations of total organic $\mathrm{P}$ by 13\%-28\%, labile organic P by $9 \%-70 \%$, moderately resistant organic $\mathrm{P}$ by $8 \%-15 \%$ and highly resistant organic P by $42 \%-56$ in the $0-5 \mathrm{~cm}$ soil layer $\%$ compared to the CT treatments. However, in the 5-20 cm soil layer, the NT treatments significantly decreased the concentrations of labile organic P, moderately resistant organic $\mathrm{P}$ and highly resistant organic $\mathrm{P}$ by $15 \%-44 \%, 11 \%-47 \%$ and $44 \%-80 \%$.
In general, significant negative correlations between labile organic $\mathrm{P}$ across all depths with rice grain yield of 2009 and P uptake were only observed in NT0 and CT0 (Table 4). Moreover, no significant correlation was found between other organic $\mathrm{P}$ fractions with rice grain yield and $\mathrm{P}$ uptake across soil layers under different treatments.

\section{Discussion}

\subsection{Crop grain yield and P uptake}

In the present study, under fertilization conditions, seed yields of rape ranged from 1948 to $2171 \mathrm{~kg} \mathrm{ha}^{-1}$ for NT and from 1925 to $2264 \mathrm{~kg} \mathrm{ha}^{-1}$ for CT during the growing seasons of 2007-2009; grain yields of rice varied from 7830 to $9307 \mathrm{~kg} \mathrm{ha}^{-1}$ for NT and from 8807 to $9453 \mathrm{~kg} \mathrm{ha}^{-1}$ for CT. 
Table 3. Concentrations of soil organic $\mathrm{P}$ fractions $\left(\mathrm{mg} \mathrm{kg}^{-1}\right)$ under different treatments after 3 years of rape-rice rotation

\begin{tabular}{|c|c|c|c|c|c|c|c|c|c|}
\hline \multirow{2}{*}{ Treatments } & \multicolumn{3}{|c|}{ Labile organic $P$} & \multicolumn{3}{|c|}{ Moderately labile organic $P$} & \multicolumn{3}{|c|}{ Moderately resistant organic $P$} \\
\hline & $0-5 \mathrm{~cm}$ & $5-10 \mathrm{~cm}$ & $10-20 \mathrm{~cm}$ & $0-5 \mathrm{~cm}$ & $5-10 \mathrm{~cm}$ & $10-20 \mathrm{~cm}$ & $0-5 \mathrm{~cm}$ & $5-10 \mathrm{~cm}$ & $10-20 \mathrm{~cm}$ \\
\hline NT0 & $\begin{array}{l}7.0 \mathrm{aAB} \\
(0.6)\end{array}$ & $\begin{array}{l}4.5 \mathrm{bC} \\
(0.4)\end{array}$ & $\begin{array}{l}5.2 \mathrm{bC} \\
(0.7)\end{array}$ & $\begin{array}{l}220 \mathrm{aA} \\
(12)\end{array}$ & $\begin{array}{l}181 \mathrm{bA} \\
(12)\end{array}$ & $\begin{array}{l}155 \mathrm{cA} \\
(14)\end{array}$ & $\begin{array}{l}27.5 \mathrm{aA} \\
(1.2)\end{array}$ & $\begin{array}{l}16.6 \mathrm{bC} \\
(1.8)\end{array}$ & $\begin{array}{l}15.1 \mathrm{bB} \\
(2.5)\end{array}$ \\
\hline СТ0 & $\begin{array}{l}6.4 \mathrm{aC} \\
(0.5)\end{array}$ & $\begin{array}{l}6.5 \mathrm{aB} \\
(0.6)\end{array}$ & $\begin{array}{l}6.0 \mathrm{aB} \\
(0.6)\end{array}$ & $\begin{array}{l}203 \mathrm{aA} \\
(20)\end{array}$ & $\begin{array}{l}147 \mathrm{bA} \\
\text { (11) }\end{array}$ & $\begin{array}{l}130 \mathrm{bA} \\
(21)\end{array}$ & $\begin{array}{l}25.5 \mathrm{aB} \\
(2.4)\end{array}$ & $\begin{array}{l}20.2 \mathrm{bB} \\
(2.9)\end{array}$ & $\begin{array}{l}22.2 \text { abA } \\
(1.4)\end{array}$ \\
\hline NTF & $\begin{array}{l}14.3 \mathrm{aA} \\
(1.2)\end{array}$ & $\begin{array}{l}6.3 \mathrm{bB} \\
(0.7)\end{array}$ & $\begin{array}{l}6.2 \mathrm{bB} \\
(0.5)\end{array}$ & $\begin{array}{l}225 \mathrm{aA} \\
(21)\end{array}$ & $\begin{array}{l}152 \mathrm{bA} \\
(13)\end{array}$ & $\begin{array}{l}140 \mathrm{bA} \\
(14)\end{array}$ & $\begin{array}{l}27.7 \mathrm{aA} \\
(3.1)\end{array}$ & $\begin{array}{l}22.6 \mathrm{aB} \\
(3.1)\end{array}$ & $\begin{array}{l}14.1 \mathrm{cB} \\
(1.0)\end{array}$ \\
\hline CTF & $\begin{array}{l}8.4 \mathrm{aB} \\
(0.9)\end{array}$ & $\begin{array}{l}8.3 \mathrm{aA} \\
(0.7)\end{array}$ & $\begin{array}{l}7.3 \mathrm{bA} \\
(0.7)\end{array}$ & $\begin{array}{l}187 \mathrm{aA} \\
(17)\end{array}$ & $\begin{array}{l}161 \mathrm{bA} \\
(14)\end{array}$ & $\begin{array}{l}147 \mathrm{bA} \\
(16)\end{array}$ & $\begin{array}{l}24.1 \mathrm{aB} \\
(3.4)\end{array}$ & $\begin{array}{l}25.1 \mathrm{aA} \\
(4.1)\end{array}$ & $\begin{array}{l}20.4 \mathrm{bA} \\
(4.6)\end{array}$ \\
\hline \multicolumn{10}{|c|}{ Analysis of variance } \\
\hline $\mathrm{F}$ & ** & $* *$ & * & ns & ns & ns & ns & ns & ns \\
\hline $\mathrm{T}$ & $* *$ & $* *$ & $* *$ & ns & ns & ns & * & * & * \\
\hline $\mathrm{F} \times \mathrm{T}$ & ns & ns & ns & ns & ns & ns & ns & ns & ns \\
\hline
\end{tabular}

\begin{tabular}{|c|c|c|c|c|c|c|}
\hline \multirow{2}{*}{ Treatments } & \multicolumn{3}{|c|}{ Highly resistant organic $P$} & \multicolumn{3}{|c|}{ Total organic $\mathrm{P}$} \\
\hline & $0-5 \mathrm{~cm}$ & $5-10 \mathrm{~cm}$ & $10-20 \mathrm{~cm}$ & $0-5 \mathrm{~cm}$ & $5-10 \mathrm{~cm}$ & $10-20 \mathrm{~cm}$ \\
\hline NT0 & $\begin{array}{l}41.7 \mathrm{aA} \\
(5.1)\end{array}$ & $\begin{array}{l}25.2 \mathrm{bB} \\
(2.6)\end{array}$ & $\begin{array}{l}27.3 \mathrm{bC} \\
(2.6)\end{array}$ & $\begin{array}{l}296 \mathrm{aA} \\
(22)\end{array}$ & $\begin{array}{l}227 \mathrm{bA} \\
(26)\end{array}$ & $\begin{array}{l}202 \mathrm{bA} \\
(9.4)\end{array}$ \\
\hline СТ0 & $\begin{array}{l}26.7 \mathrm{bB} \\
(3.1)\end{array}$ & $\begin{array}{l}45.4 \mathrm{aA} \\
(4.9)\end{array}$ & $\begin{array}{l}40.0 \mathrm{aA} \\
(4.8)\end{array}$ & $\begin{array}{l}262 \mathrm{aB} \\
(32)\end{array}$ & $\begin{array}{l}219 \mathrm{bA} \\
(31)\end{array}$ & $\begin{array}{l}198 \mathrm{bA} \\
\text { (11) }\end{array}$ \\
\hline NTF & $\begin{array}{l}48.5 \mathrm{aA} \\
(4.4)\end{array}$ & $\begin{array}{l}24.9 \mathrm{bB} \\
(2.4)\end{array}$ & $\begin{array}{l}23.8 \mathrm{bC} \\
(2.0)\end{array}$ & $\begin{array}{l}325 \mathrm{aA} \\
(25)\end{array}$ & $\begin{array}{l}206 \text { bA } \\
\text { (24) }\end{array}$ & $\begin{array}{l}184 \mathrm{bA} \\
(15)\end{array}$ \\
\hline $\mathrm{CTF}$ & $\begin{array}{l}34.2 \mathrm{aB} \\
(5.1)\end{array}$ & $\begin{array}{l}36.1 \mathrm{aA} \\
(3.7)\end{array}$ & $\begin{array}{l}34.2 \mathrm{aB} \\
(1.9)\end{array}$ & $\begin{array}{l}254 \mathrm{aB} \\
(19)\end{array}$ & $\begin{array}{l}230 \text { abA } \\
(18)\end{array}$ & $\begin{array}{l}209 \mathrm{bA} \\
(19)\end{array}$ \\
\hline \multicolumn{7}{|c|}{ Analysis of variance } \\
\hline $\mathrm{F}$ & ns & ns & ns & ns & ns & ns \\
\hline $\mathrm{T}$ & $* *$ & $* *$ & $* *$ & $*$ & ns & ns \\
\hline $\mathrm{F} \times \mathrm{T}$ & ns & ns & ns & ns & ns & ns \\
\hline
\end{tabular}

Different small letters in a line at different soil depths indicate significant difference at the $5 \%$ level. Different capital letters in a column mean significant difference at the $5 \%$ level between treatments. Values in brackets are standard deviation. NT0, no-tillage with no fertilizer; CT0, conventional tillage with no fertilizer; NTF, no-tillage with application of chemical fertilizer; CTF, conventional tillage with application of chemical fertilizer; F, application of chemical fertilizer; T, tillage practices; ns, not significant; ${ }^{*}, p<0.05 ; * *, p<0.01$.

The yields fall within crop yields reported by Huang et al. (2011) in the same region. They indicated that rape seed yields varied from 1540 to $1790 \mathrm{~kg} \mathrm{ha}^{-1}$ for $\mathrm{CT}$ and from 1520 to $2120 \mathrm{~kg} \mathrm{ha}^{-1}$ for NT, and rice grain yields ranged from 8380 to $11250 \mathrm{~kg} \mathrm{ha}^{-1}$ for $\mathrm{CT}$ and 8700 to $11120 \mathrm{~kg} \mathrm{ha}^{-1}$ for NT. Moreover, the yield difference of crop was not significant between $\mathrm{CT}$ and NT (Table 1), meaning that greater returns of crop residues to the soil surface under NT. Our results were consistent with the findings of Huang et al. (2011) who found similar yields of rice and rape between CT and NT in rice-rape cropping system of central China. Similar results were also observed by other researchers (Qin et al, 2010, But, Jiang and Xie (2009) reported that NT increased rice grain yields compared to CT because of improvement of soil physico-chemical properties. Moreover, in some cases, lower crop yields of rice-based systems under NT were also observed 
(Sharma et al., 2005; Xie et al., 2007) reviewed the effect of conservation tillage on crop yields in China and found that in most cases, conservation tillage does not reduce crop yield, but in some cases, it decreases crop yield. They surmised that the difference in crop yields between tillage systems might depend on different climates, soil fertility or the duration time of no-tillage. In the present study, we found that crop yields of the rape-rice rotation under NT did not increase with time across three years (Table 1), inconsistent with Wang et al. (2001). This indirectly implied effects of climate changes across three years on crop yields. Basamba et al. (2006) indicated that the transition from CT to NT often leads to changes in crop yield during the initial years after conversion, possibly as a result of differences in nutrient immobilization/mineralization dynamics of soil, and thus they observed lower maize grain yield under NT in the first year after conversion but higher yield in the second year compared to CT. However, in the present study, the difference observed in crop yield was not significant during the first 3 years after CT conversion to NT (Table 1). Triplett and Dick (2008) analyzed crop responses to NT in the United States and suggested that three year might be required for NT systems to become fully functional, when changing from CT to NT. Wang et al. (2001) reported crop yields of rice-wheat rotation systems improved with time in continuous NT paddy soils. In this system, crop yields were similar between NT and CT for the first 2 years, but by the third year of the study, NT had greater yields. Clearly, more information on short-term NT effects on crop yield is needed.

The fertilized treatments had significantly higher crop yields compared to the no fertilizer treatments (Table 1), showing a significant effect of fertilization on crop yields. The positive effect of fertilization on rice yields has been well demonstrated (Lan et al., 2012).

Similar to crop yield, tillage had no significant effect on P uptake but fertilization increased P uptake (Table 1). Our results contrast with the findings of Ibrahim et al. (2011), who reported significant decreases in $\mathrm{P}$ uptake by rape and rice under reduced tillage or NT due to more competition for $\mathrm{P}$ from weeds.

\subsection{Soil organic P fractions}

In general, the distribution of total $\mathrm{P}$, Bray-1 $\mathrm{P}$, inorganic $\mathrm{P}$ and organic $\mathrm{P}$ fractions in the NT soils was strongly stratified when compared to the $\mathrm{CT}$ soils (Tables 2 and 3). This was apparently due to reduced mixing of fertilizer P under NT (Ma et al., 2003), possibly increasing quantities of $\mathrm{P}$ fractions on the soil surface, or due to greater crop residues being returned to the soil, which increases $\mathrm{P}$ concentration by decreasing the adsorption of $\mathrm{P}$ to mineral surfaces (Jiang and Xie, 2009).

Higher total $\mathrm{P}$, inorganic $\mathrm{P}$ and Bray-1 $\mathrm{P}$ on the soil surface $(0-5 \mathrm{~cm})$ for the NT treatments than the CT treatments (Table 2) has been previously well confirmed (Jiang and Xie, 2009; Ma et al., 2003). This is consistent with accumulation of crop resides near the soil surface and a lack of mixing of soil and fertilizer (Ma et al., 2003). Greater total $P$, inorganic $P$ and Bray-1 P under NT implied that to obtain the same crop yields to CT, less P may need to be applied under NT due to greater $\mathrm{P}$ availability resulting in greater diffusion rate of $\mathrm{P}$ towards the plant root. In addition, although CT had lower availability of $\mathrm{P}$ on the soil surface compared to NT, lower $\mathrm{N}$ fertilizer loss as previously reported by Zhang et al. (2011), and better control of weeds (results not shown) under CT might possibly explain why there was no significant difference in crop yields between NT and CT in the present study (Table 1).

Fertilization significantly enhanced acid phosphatase activity in the $0-5 \mathrm{~cm}$ soil layer, possibly reflecting positive effects of fertilization on total inorganic $\mathrm{P}$ and Bray-1 P (Table 2). This agrees with the findings of Lee et al. (2004) Under NT, there was a concentration gradient of acid phosphatase activity across all depths (Table 2), generally reflecting soil organic matter stratification under NT that was reported in our previous study (Zhang et al., 2011). We observed significantly greater acid phosphatase activity on the 
Table 4. Linear correlations between soil organic $\mathrm{P}$ fraction with rice grain yield and $\mathrm{P}$ uptake of 2009. Only significant $(p<0.05)$ correlations were shown.

\begin{tabular}{|c|c|c|c|c|c|c|c|c|c|}
\hline \multirow[t]{2}{*}{ Items } & \multirow[t]{2}{*}{ Depth } & \multicolumn{4}{|c|}{ Rice grain yield } & \multicolumn{4}{|c|}{ Rice P uptake } \\
\hline & & NT0 & CT0 & NTF & CTF & NT0 & CT0 & NTF & CTF \\
\hline \multirow[t]{3}{*}{ Total organic $\mathrm{P}$} & $0-5 \mathrm{~cm}$ & & $-0.68^{*}$ & & & & & & \\
\hline & $5-10 \mathrm{~cm}$ & & & & & & & & \\
\hline & $10-20 \mathrm{~cm}$ & & & & & & & & \\
\hline \multirow[t]{3}{*}{ Labile organic $\mathrm{P}$} & $0-5 \mathrm{~cm}$ & $-0.85^{* *}$ & $-0.80^{* *}$ & & & $-0.81^{* *}$ & $-0.82^{* *}$ & & \\
\hline & $5-10 \mathrm{~cm}$ & $-0.82^{* *+}$ & $-0.87^{* *}$ & & & $-0.78^{*}$ & $-0.77^{*}$ & & \\
\hline & $10-20 \mathrm{~cm}$ & $-0.83^{* *}$ & $-0.82^{* *}$ & & & $-0.80^{* *}$ & $-0.80^{* *}$ & & \\
\hline \multirow[t]{3}{*}{ Moderately organic P } & $0-5 \mathrm{~cm}$ & & & & & & & & \\
\hline & $5-10 \mathrm{~cm}$ & & & & & & & & \\
\hline & $10-20 \mathrm{~cm}$ & & & & & & & & \\
\hline \multirow[t]{3}{*}{ Moderately organic $\mathrm{P}$} & $0-5 \mathrm{~cm}$ & & & & & & & & \\
\hline & $5-10 \mathrm{~cm}$ & & & & & & & & \\
\hline & $10-20 \mathrm{~cm}$ & & & & & & & & \\
\hline \multirow[t]{3}{*}{ Highly resistant organic $P$} & $0-5 \mathrm{~cm}$ & $-0.67^{*}$ & & & & & $-0.67^{*}$ & & \\
\hline & $5-10 \mathrm{~cm}$ & & & & & & & & \\
\hline & $10-20 \mathrm{~cm}$ & & & & & & & & \\
\hline
\end{tabular}

NT0, no-tillage with no fertilizer; CT0, conventional tillage with no fertilizer; NTF, no-tillage with application of chemical fertilizer; CTF, conventional tillage with application of chemical fertilizer; *, $p<0.05 ; * *, p<0.01 ; \mathrm{n}=9$.

soil surface under NT compared to CT, consistent with Huang et al. (2012). This may be due to higher soil organic matter levels and higher amounts of fungi on the soil surface under NT (Huang et al., 2012).

In the present study, greater total organic $\mathrm{P}$ on the soil surface under NT compared to CT (Table 3), reflects greater amount of crop residues returned to the NT soils, and a reduction in rate of decomposition often reported for crop residues left at the soil surface in NT systems. Our result contrasts with the result of Huang and Yuan (1994) who reported a decrease in organic $\mathrm{P}$ under NT paddy soils. Moreover, we observed an increase in organic $\mathrm{P}$ resulting from an increase in abile organic $\mathrm{P}$ (Table 3), consistent with Tiecher et al. (2012) who reported that increases in organic P forms under NT occurred in labile organic and moderately llabile P. We did not observe a significant effect of fertilization on total organic $\mathrm{P}$ (Table 3). This agrees with the findings of Zhang et al. (2006) However, Lan et al. (2012) and Lee et al. (2004) reported that fertilizer application resulted in accumulation of organic $\mathrm{P}$ in paddy soils.

Zhang et al. (1994) reported that fertilizer P plays an important role in promoting the formation of organic P fractions on paddy soils. However, our results showed an increase in labile organic $\mathrm{P}$ and lack changes in total inorganic and organic $\mathrm{P}$ due to application of fertilizer (Table 3). This implies that fertilization had no effect on the concentration of organic P but increased the availability of P in soil. Our results contrasts with Zhang et al. (2006) who reported no significant effect of fertilization on $\mathrm{NaHCO}_{3}$ extractable organic $\mathrm{P}$ in a calcareous paddy soil, but are in agreement with Lan et al. (2012) who 
found application of chemical fertilizers significantly enhanced concentrations of labile organic $\mathrm{P}$ and total organic $\mathrm{P}$ concentration. Increased labile organic $\mathrm{P}$ due to addition of $\mathrm{P}$ is possibly explained by inorganic $\mathrm{P}$ from addition of $\mathrm{P}$ fertilizer being taken up by microbes to form organic $\mathrm{P}$ compounds present in microbial detritus that acts as a source of labile organic $\mathrm{P}$ in soil (Lee et al., 2004).

It has been reported that crop yield is limited by low soil $\mathrm{P}$ availability in subtropical areas (Bao, 2000). In the present study, negative correlations were observed between labile organic $\mathrm{P}$ with rice grain yield of 2009 and P uptake in the no fertilizer treatments (Table 4). This showed that labile organic $P$ through mineralization acts as a rice-available $\mathrm{P}$ source in unfertilized soils and an adequate supply of $\mathrm{P}$ from fertilizers is essential for maintaining crop productivity in paddy soils with low $\mathrm{P}$ availability in central China. In the fertilized treatments, the rice grain yield was not significantly related to soil organic $\mathrm{P}$ fractions, indicating that fertilization met the $\mathrm{P}$ requirement of rice plants.

In the present study, the continuous 3-year adoption of NT significantly increased soil organic P fractions on the soil surface $(0-5 \mathrm{~cm})$ with the exception of moderately labile organic P (Table 3 ). This is mainly because of higher returns of crop residues to the soil surface under NT. In contrast, the continuous adoption of NT decreased soil organic P fractions in the lower soil layer $(5-20 \mathrm{~cm})$ except moderately labile organic $\mathrm{P}$, reflecting the mixing of soil and crop residues under CT. Our results indicate that short-term NT (3 years of CT conversion to NT) only changes distribution of soil $P$ in the $0-20 \mathrm{~cm}$ soil layer, does not increase soil organic $\mathrm{P}$ concentrations and only enhanced $\mathrm{P}$ availability on the soil surface (Tables 2 and 3). Influences of tillage practices on soil organic $\mathrm{P}$ fractions depend not only on the soil physical disturbance and the distribution of crop residues, but also on the duration of tillage practices. Quan et al. (2005) reviewed effects of the duration of NT on soil fertility of paddy fields, and indicated that short-term NT only improved soil physical properties and did not enhance soil $\mathrm{P}$ concentration. Moreover, long-term tillage effect includes the effect of changes in physical, chemical and biological soil properties after several years of conversion to NT (Quan et al., 2005). However, the long-term impact of NT on soil organic P fractions in central China is largely unknown, and thus further research is needed to quantify this impact.

\section{Conclusions}

Agricultural management practices affected crop yield, P uptake and soil organic P fractions. Fertilization significantly increased crop yields and $P$ uptake but tillage practices did not affect the yields and $\mathrm{P}$ uptake. Fertilization significantly enhanced acid phosphatase activity and concentrations of total P, Bray-1 P, total organic P, labile organic P in the $0-5 \mathrm{~cm}$ soil layer but did not affect other organic $\mathrm{P}$ fractions. Compared to $\mathrm{CT}$, NT had greater concentrations of organic $\mathrm{P}$ fractions in the $0-5 \mathrm{~cm}$ soil layer but lower concentrations in the 5-20 cm soil layer, suggesting that short-term NT only improved $\mathrm{P}$ availability on the soil surface.

\section{Acknowledgements}

This research was funded by the National Technology Project for High Food Yield, China (2011BAD16B02), National Natural Science Foundation of China (31100319), Program for Changjiang Scholars and Innovative Research Team in University of China (IRT1247) and Fundamental Research Funds for the Central Universities (2013PY106). We would like to express our utmost appreciation Dr. Farooq Shah for a pertinent review. We are grateful to María de la Luz Mora (Editor-in-Chief), Alex Seguel (Managing editor)and anonymous reviewers for the constructive comments on the manuscript. 


\section{References}

Bao, S.D. 2000. Methods Analysis for Soil Science and Agricultural Chemistry. Beijing: Chinese Agricultural Press.

Basamba, T.A., Barrios, E., Amézquita, E., Rao, I.M., Singh, B.R. 2006. Tillage effects on maize yield in a Colombian savanna oxisol: soil organic matter and P fractions. Soil Till. Res. 91, 131-142.

Bowman, R.S., Cole, C.V. 1978. An exploratory method for fractionation of organic phosphorus from grassland soils. Soil Sci. 125, 95-101.

Bray, R.H, Kurtz, L.T. 1945. Determined of total, organic and available forms of P in soils. Soil Sci. $59,39-45$.

Conte, E., Anghinoni, I., Rheinheimer, D.S. 2003. Phosphorus accumulation fractions in a clayey oxisol in relation to phosphate doses under notillage. Revista Brasileira De Ciencia Do Solo. 27, 893-900.

Dalal, R.C. 1997. Soil organic phosphorus. Adv. Agron. 29, 83-117.

Daniel, T.C., Sharpley, A.N., Lemunyon, J.L. 1998. Agricultural phosphorus and eutrophication - a symposium overview. J. Environ. Qual. 27, 251257.

Huang, J.G., Yuan, L. 1994. Effects of tillage on P status in paddy soils. J. Southwest Agri. Uni. 16, 475-477.

Huang, M., Zou, Y.B., Feng, Y.H., Cheng, Z.W., Mo, Y.L., Ibrahim, M., Xia, B., Jiang, P. 2011. Notillage and direct seeding for super hybrid rice production in rice-oilseed rape cropping system. Eur. J. Agron. 34, 278-286.

Huang, M., Zou, Y., Jiang, P., Xia, B., Feng, Y.H., Cheng, Z.W., Mo, Y.L. 2012. Effect of tillage on soil and crop properties of wet-seeded flooded rice. Field Crops Res. 129, 28-38.

HPBS (Hubei Provincial Bureau of Statistics). 2010. Hubei Rural Statistical Yearbook. China Statistical Press, 35-48. (in Chinese)

Ibrahim, M., Yamin, M., Sarwar, M.G., Anayat, A., Habib, F., Ullah, S., Saif-ur-Rehman. 2011. Tillage and farm manure affect root growth and nutrient uptake of wheat and rice under semi-arid conditions. Appl. Geochem. 26, S194-S197.

Jiang, X.J., Xie, D.T. 2009. Combining ridge with no-tillage in lowland rice-based cropping system: long-term effect on soil and rice yield. Pedosphere. $19,515-522$.

Lan, Z.M., Lin, X.J., Wang, F., Zhang, H., Chen, C.R. 2012. Phosphorus availability and rice grain yield in a paddy soil in response to long-term fertilization. Biol. Fertil. Soils. 48, 579-588.

Lee, C.H., Lee, I.B., Kim, P.J. 2004. Effects of longterm fertilization on organic phosphorus fraction in paddy soil. Soil Sci. Plant Nutr. 50, 485-491.

Ma, H.L., Gao, M., Wei, C.F. 2003. Effect of agronomic management on forms of inorganic phosphorus in purple paddy soil. Soils. 35, 24-254.

Murphy, J., Riley, J.P. 1962. A modified single solution method for the determination of phosphate in natural waters. Anal. Chim. Acta 27, 31-36.

Qin, J., Hu, F., Li, D., Li, H., Lu, J., Yu, R. 2010. The effect of mulching, tillage and rotation on yield in non-flooded compared to flooded rice production. J. Agron. Crop Sci. 196, 397-406.

Quan, G.M., Zhang, J.E., Yan, H.C., Xu, R.B. 2005. Review on influences of no-tillage on soil fertility of paddy field. Chin. Agri. Sci. Bull. 21, 266-269, 278. 
Selles, F., Kochhann, R.A., Denardin, J.E., Zentner, R.P., Faganello, A. 1997. Distribution of phosphorus fractions in a Brazilian Oxisol under different tillage systems. Soil Till. Res. 44, 23-34.

Sharma, P., Tripathi, R.P., Singh, S. 2005. Tillage effects on soil physical properties and performance of rice-wheat-cropping system under shallow water table conditions of Tarai, Northern India. Eur. J. Agron. 23, 327-335.

Sharpley, A.N. 1985. Phosphorus cycling in unfertilized and fertilized agricultural soils. Soil Sci. Soc. Am. J. 49, 905-911.

Tabatabai, M.A., Bremner, J.M. 1969. Use of p-nitrophenyl phosphate for assay of soil phosphatase activity. Soil Biol. Biochem. 1, 301-307.

Tiecher, T., dos Santos, D.R., Calegari, A. 2012. Soil organic phosphorus forms under different soil management systems and winter crops in a long term experiment. Soil Till. Res. 124, 57-67.

Triplett, G.B.Jr., Dick, W.A. 2008. No-tillage crop production: a revolution in agriculture!. Agron. J. 100, S-153-S-165.
Wang, C.Q., Wei, C.M., Li, T.Q., Sun, F.Q. 2001. Effect of different zero tillage on the crop yield and soil property. J. Sichuan Agri. Univ. 19(2), 152-155.

Xie, R.Z., Li, S.K, Li, X.J., Jin, Y.Z., Wang, K.R., Chu, Z.D., Gao, S.J. 2007. The analysis of conservation tillage in China - conservation tillage and crop production: reviewing the evidience. Sci. Agri. Sinica. 40, 1914-1924.

Zhang, J.S., Zhang, F.P., Yang, J.H., Wang, J.P., Cai, M.L., Li, C.F., Cao, C.G. 2011. Emissions of $\mathrm{N}_{2} \mathrm{O}$ and $\mathrm{NH}_{3}$, and nitrogen leaching from direct seeded rice under different tillage practices in central China. Agri. Ecosyst. Environ. 140, 164-173.

Zhang, Q., Wang, G.H., Feng, Y.K., Sun, Q.Z., Wit, C., Dobermann, A. 2006. Changes in soil phosphorus fractions in a calcareous paddy soil under intensive rice cropping. Plant Soil. 288, 141-154.

Zhang, Y.S., Werner, W., Scherer, H.W., Sun, X. 1994. Effect of organic manure on organic phosphorus fractions in two paddy soils. Biol.Fertil.Soils.17, 64-68. 